\title{
Using Artificial Intelligence for Quantifying Strategic Business-IT Alignment
}

\author{
Bassel DIAB \\ The Bucharest University of Economic Studies, Romania \\ Basseljdiab@gmail.com
}

This paper aims to test an artificial model and a calculator the author developed based on deep learning, Neural Networks, and machine learning, Random Forest. The "Diab BITA Model" and the "Diab Calculator" are generated to enable organizations, of any size and in any industry, of calculating the value of Strategic Business-IT Alignment (BITA) following a scale of 7 degrees. Principally, the same sample of one of his previous papers is addressed in which top managers subjectively assessed the BITA maturity; the current paper targets to empirically prove the accuracy of managers' perceptions using both the model and the calculator. Findings show an $89 \%$ accuracy rate in estimating those organizations' BITA levels using the model and $92 \%$ using the calculator.

Keywords: Deep Learning, Machine Learning, Diab BITA Model, Diab Calculator DOI: $10.24818 /$ issn14531305/25.1.2021.05

1 Introduction

Till date, there is no consent on one definition of Strategic Business-IT Alignment. Henderson \& Venkatraman (1993) simply define it as the integration level between business strategy \& infrastructure and IT strategy \& infrastructure [1]. A wide literature is available; however, BITA is still neither found on particular theories nor practically validated. Also, a serious gap actually exists in literature although BITA is one of the top challenges facing organizations in the contemporary business world. On the other hand, some strategic alignment models have been developed and tested in order to evaluate strategic alignment in an organization. For instance, models include SAM (Strategic Alignment Model), the Generic Framework Alignment Model and SAMM (Strategic Alignment Maturity Model). Moreover, most of the investigations which have studied the enablers and inhibitors of BITA focus mainly on the cooperative work of business and IT executives, based on various factors linking business with IT technically and "businessically" like "senior executives support for IT" or "IT understands the business". Furthermore, the Control Objectives for Information and Related Technology (COBIT), being one of the leading IT governance frameworks, is fundamentally concerned with BITA. "The COBIT business orientation includes linking business goals with its IT infrastructure by providing various maturity models and metrics that measure the achievement while identifying associated business responsibilities of IT processes" (Simplilearn, 2020) [2]. Oppermann (2019) defines deep learning to be " $a$ subset of Machine Learning, which on the other hand is a subset of Artificial Intelligence... Deep Learning, on the other hand, is just a type of Machine Learning, inspired by the structure of a human brain... deep learning uses a multi-layered structure of algorithms called neural networks" [3] (Fig. no. 1). 


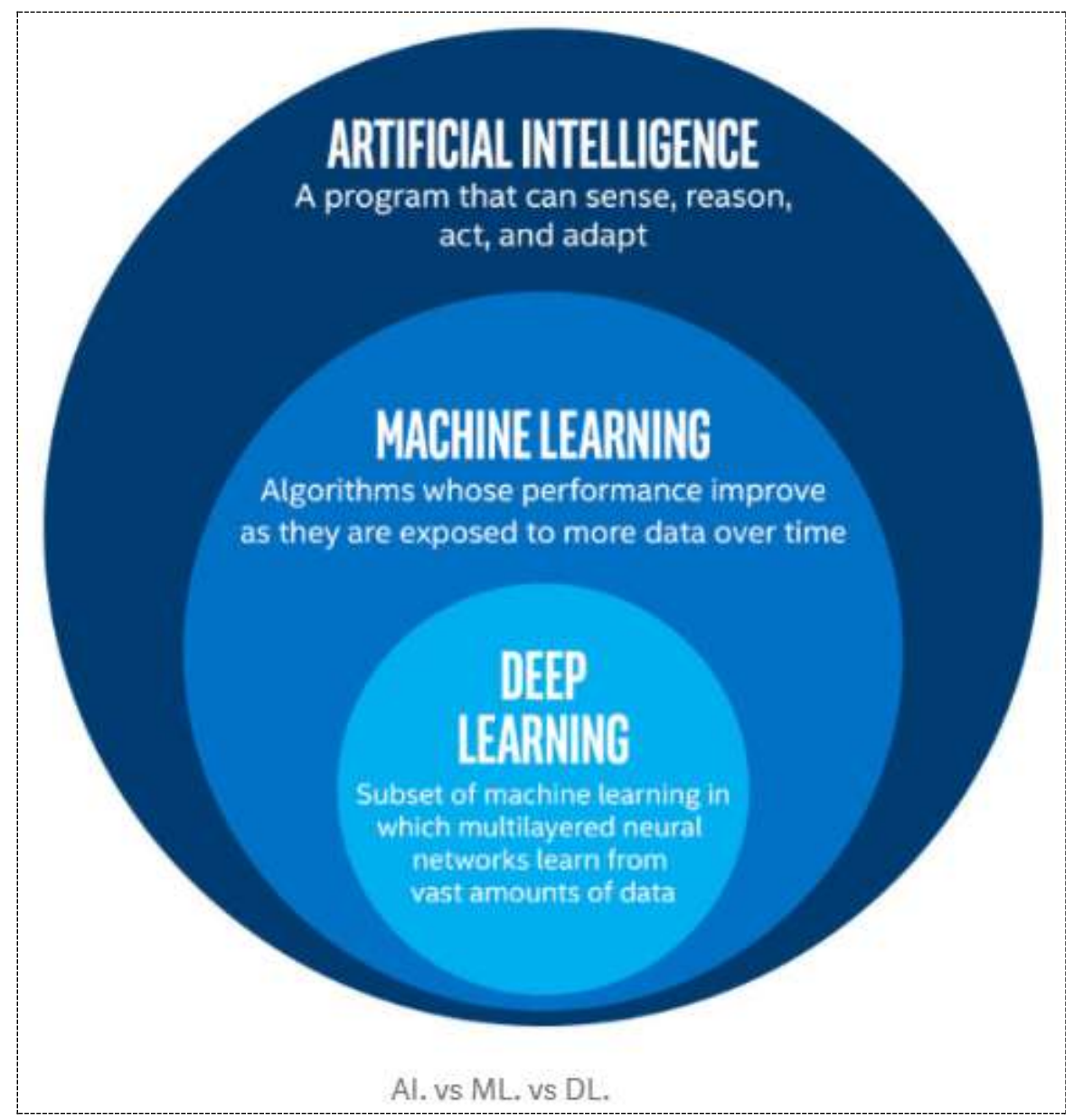

Fig. 1. AI. vs ML. vs DL. [3]

\section{Motivation for this Study}

In his $\mathrm{PhD}$ research, one of the core motivations of the author is to provide selfdeveloped metrics written in simple words and expressions so that top managements could be able to numerically measure the level and maturity of BITA at their organizations. Besides, this work is expected to facilitate the communication of BITA assessment results as well as its discussion with subordinates or other departments (i.e. IT, finance, production, etc.), considering that one of the major problems encountering business and IT people is their mutual communication. They either engage in weak communication practices or speak two different, business and technical, languages [4]. Equally, cultural and social drivers falling under organizational culture and communication are incorporated; this contributes to narrowing the gap found in the majority of other BITA models which neglect to a far extent such types of factors.
Due to time and budget constraints, the author bootstrapped the primary data originally collected and applied SPSS, Neural Networks, and RStudio programming language, Random Forest. He came out with a model comprising 31 independent variables and only one dependent variable which is BITA. The model consists of four modules which intend to evaluate the points of view of top managements based on a 7-point Likert scale. The first three modules, organizational communication-organizational cultureorganizational performance, include 8 indicators each and the fourth module, BITA, includes 7 indicators. The ultimate goal is to convert raw data into a scientific instrument governing the contribution of those independent variables to BITA maturity. To illustrate for this paper's benefit, the author is going to focus on SPSS he used and Neural Networks, Multilayer Perceptron, he adopted as a modeling tool to minimize error and to 
ensure accurate predictions for a better decision making.

The model was trained with $70 \%$ of data $(703$ observations) and the remaining $30 \%$ of data (296 observations) was tested using that model which eventually reached a $3.4 \%$
"Percent Incorrect Predictions" which implies a $96.6 \%$ accuracy rate. Therefore, the model was successful in predicting the BITA level of $96.6 \%$ of the surveyed organizations (Fig. no. 2).

\begin{tabular}{|c|c|c|}
\hline \multicolumn{3}{|c|}{ Model Summary } \\
\hline \multirow[t]{4}{*}{ Training } & Cross Entropy Error & 71.742 \\
\hline & $\begin{array}{l}\text { Percent Incorrect } \\
\text { Predictions }\end{array}$ & $3.3 \%$ \\
\hline & Stopping Rule Used & $\begin{array}{l}1 \text { consecutive } \\
\text { step(s) with no } \\
\text { decrease in } \\
\text { error }^{\text {a }}\end{array}$ \\
\hline & Training Time & $0: 00: 02.38$ \\
\hline \multirow[t]{2}{*}{ Testing } & Cross Entropy Error & 32.726 \\
\hline & $\begin{array}{l}\text { Percent Incorrect } \\
\text { Predictions }\end{array}$ & $3.4 \%$ \\
\hline \multicolumn{3}{|c|}{$\begin{array}{l}\text { Dependent Variable: Strategic alignment in your } \\
\text { organization is ranked as high. }\end{array}$} \\
\hline \multicolumn{3}{|c|}{$\begin{array}{l}\text { a. Error computations are based on the testing } \\
\text { sample. }\end{array}$} \\
\hline
\end{tabular}

Fig. 2. Diab BITA Model Summary (Diab, B., PhD 2020)

That excellent accuracy rate urged the author to make extra empirical testing for the novel model. He perceives this testing as a starting point prior to approving the launching of model into research and academic area by converting the model into a managerial applicative tool, the "Diab Calculator" (Appendix 1). The calculator is going to be built in the format of a mobile application with open-access rights to render it much smooth for end-users. In this application, the potential user (e.g., GM, senior executive) is requested to insert as input his/her ranking of 31 independent variables covering major organizational aspects (i.e., organizational communication, organizational culture, organizational performance \& strategic alignment) so as the application computes the estimated importance of each variable. The overall "Estimated Importance" could reach a maximum score equal to "1" (Appendix 2).

Simultaneously, the author sought to experiment the model and calculator while verifying the potency of transparent and unbiased managers to assess the level of BITA in their organizations depending on their own specific metrics which could be one, more than one or even without having any. Details are explained in the research methodology section.

\section{Literature Review}

Iansiti and Lakhani (2020) argue that companies all over the world are forecasted to spend $\$ 98$ billion on artificial intelligence (AI) in 2023 while its spending was $\$ 38$ billion in 2019 [5]. The Business World IT, a website specialized in innovative technologies, defines the role of $\mathrm{AI}$ in business as it "simply involves the use of intelligent computer software with human-like capabilities to boost revenue, improve customer experience, increase productivity and efficiency, and drive business growth and transformation" (2020) [6]. For instance, Larry Page asserts that "Artificial intelligence would be the ultimate version of Google. The ultimate search engine that would understand everything on the web". $\mathrm{He}$ adds that "Companies that do not want to miss out on 
the revolutionary future of business must, inevitably, embrace the use of AI as a guiding light". Also, AI has extensively become adopted and implemented in numerous business sectors mainly the financial services one. Forrester, the research group, concludes that "About two-thirds of finance firms have implemented or are adding AI in areas from customer insights to IT efficiencies" (Financial Times, 2020) [7]. Other sectors include but not limited to high tech and communications, transport and logistics, travel and tourism, healthcare, energy and resources, and education.

However, a business must redesign its overall corporate business strategy (i.e. processes, tasks, human resources, etc.) in a way allowing it to adopt AI successfully. To give a clear example, a speaker in the FT forum explains that her company adopted a tool "to eradicate inefficiencies in manufacturing processes" [7]. AI completely met the expectations and goals set, but "the companies were not ready to act as their entire workflows would have to change" (2020) [7]. As well, the corporate IT strategy must be subject to an entire overhaul to properly operate within the AI environment. As a result, both of organizational business and IT strategies \& infrastructures must be perfectly integrated so as to cope with the adoption of AI as well as to avoid any potential confusing and fast changes.

Furthermore, some scholars argue that the current business world is controlled by Data. Hence, "The current era of data has stimulated the rise of many applications of artificial intelligence in business including data analytics, personalized business offerings and automation" (The Business World IT, 2020). Nevertheless, the author could not find theoretical nor empirical attempts relying on $\mathrm{AI}$ and leading to practical models or instruments that are capable of measuring the maturity level of BITA. Therefore, this paper could serve to the best of his knowledge as the first managerial tool enabling companies to benefit from the never stopping increase in the amount of data available to train and test deep learning and machine learning algorithms to fulfill this aim.

\section{Research Methodology}

To achieve the purpose of this paper, the author looks forward to proving the authenticity of the model and calculator through randomly targeting the same sample formed by 250 big organizations and SMEs which participated, in year 2019, in his paper titled "Modeling the impact of information system on organizational culture and Business-IT strategic alignment" (Informatica Economica, vol. 23, no.3). In one part of that paper, participants were asked to rank the leading enablers boosting the maturity of BITA in Small and Medium-sized Enterprises (SMEs) compared to large enterprises operating in Lebanon. Also, they graded the strategic alignment level following a 6-point Likert scale. Results yielded in a classification of these enablers based on their potential influence on the BITA level.

A sample of the data set utilized to study the correlation between BITA and the four enablers is presented in the table below (Table no. 1) [8].

The fast deteriorating economic and monetary situations in Lebanon have been leading a big number of organizations to cutting back their operations or to shutting down. Therefore, the author first contacted all of the 250 companies and sent them pre-notifications; only 89 responses were received expressing their interest to participate in the new survey where 64 were SMEs and 25 were big organizations. In this paper, a 7-point Likert scale was used while the above-mentioned paper used a 6point Likert scale. Thus, level " 6 " or " 7 " of strategic alignment in the current study was classified as equivalent to level " 6 " in the previous paper. In exchange, the participated organizations were given a word to keep them up-to-date whenever future amendment or further development is applied on the model and calculator. In addition, the author promised them to have an endless right of using it and its subsequent modifications upon request. 
To test the goodness-of-fit of the model, a Chi-square $(\chi 2)$ test was done and concluded that 356.000>> 30.6 with 15 degree of freedom at a critical value of 0.01 . This means that the model greatly fits the data.

Table 1. Data set (Source: Informatica Economica 2019)

\begin{tabular}{|c|}
\hline Respondent \\
\hline 1 \\
\hline 2 \\
\hline 3 \\
\hline 4
\end{tabular}

\section{Findings and Interpretations}

The Cronbach's Alpha was calculated to test the reliability of the artificial model and its constructs. Cronbach's Alpha of the model is $0.96>0.70$ which means it is reliable and valid.

Figure no. 3 illustrates the output of applying the model in the current study.

54 observations representing $60.7 \%$ of the overall sample were trained and 35 observations representing $39.3 \%$ of sample were tested. The "Percent Incorrect Predictions" was $11.4 \%$. For example, the model precisely projected a BITA level " 6 " in 14 organizations out of 15 ones, a level " 5 " in 10 organizations out of 11 ones, a level " 4 " in 2 organizations out of 3 ones, a level " 3 " in 5 organizations out of 5 ones, but it missed level " 2 " in the one and only organization. This is in comparison with the preceding judgments of the executives.

Also, the top 5 important independent variables concluded by SPSS are as follows: 1- Strategic IT planning is formally conducted with effective business executive's involvement $=100 \%$.
2- IS increases the readiness of the organization for institutionalization, transition to professional management and true corporate governance $=38.4 \%$.

3- IS supports the organization in adapting to fast changes in business environment (e.g. major changes/switch to a new hierarchy) $=$ $36.6 \%$.

4- IS supports executives in motivating employees to estimate the role of culture in business (e.g. knowledge sharing) $=36.3 \%$.

5- The training on IS provided by top management enables employees to optimizing the system's full capabilities $=35.0 \%$.

The factor which is judged to be the most influential promoter of BITA level is the "Business involvement in strategic IT planning"; it is one of the variables included in the strategic alignment module. Whereas the least affecting factor is the "Impact of information system on the quality of information shared" which is, in turn, an organizational communication module's variable. 


\section{Classification}

\begin{tabular}{|c|c|c|c|c|c|c|c|}
\hline \multirow[b]{2}{*}{ Sample } & \multirow[b]{2}{*}{ Observed } & \multicolumn{6}{|c|}{ Predicted } \\
\hline & & 2 & 3 & 4 & 5 & 6 & $\begin{array}{l}\text { Percent } \\
\text { Correct }\end{array}$ \\
\hline \multirow[t]{6}{*}{ Training } & 2 & 0 & 2 & 3 & 0 & 0 & $0.0 \%$ \\
\hline & 3 & 0 & 3 & 2 & 0 & 0 & $60.0 \%$ \\
\hline & 4 & 0 & 3 & 5 & 0 & 0 & $62.5 \%$ \\
\hline & 5 & 0 & 0 & 0 & 20 & 0 & $100.0 \%$ \\
\hline & 6 & 0 & 0 & 0 & 0 & 16 & $100.0 \%$ \\
\hline & Overall Percent & $0.0 \%$ & $14.8 \%$ & $18.5 \%$ & $37.0 \%$ & $29.6 \%$ & $81.5 \%$ \\
\hline \multirow[t]{6}{*}{ Testing } & 2 & 0 & 1 & 0 & 0 & 0 & $0.0 \%$ \\
\hline & 3 & 0 & 5 & 0 & 0 & 0 & $100.0 \%$ \\
\hline & 4 & 0 & 1 & 2 & 0 & 0 & $66.7 \%$ \\
\hline & 5 & 0 & 0 & 1 & 10 & 0 & $90.9 \%$ \\
\hline & 6 & 0 & 0 & 0 & 1 & 14 & $93.3 \%$ \\
\hline & Overall Percent & $0.0 \%$ & $20.0 \%$ & $8.6 \%$ & $31.4 \%$ & $40.0 \%$ & $88.6 \%$ \\
\hline
\end{tabular}

Fig. 3. Output of Study (self-developed)

Consistently, data of the 89 participants were processed via the BITA calculator which is currently in its final stage of innovation. Results were astonishing as it demonstrated to be successful in estimating the BITA level at 82 organizations with $92 \%$ accuracy rate. Thus far, the calculator showed that 7 senior managers, representing the missed $8 \%$, overestimated the maturity of their organizations strategic alignment. 4 managers assessed BITA to be " 6 " while the calculator stated it should be " 5 " and 3 managers assessed BITA as " 6 " but the calculator stated "4". Additionally, 27 executives underestimated the BITA maturity in their businesses; for example, 13 managers evaluated BITA to be " 5 " while it should be " 6 " and 3 managers to be " 3 " while the calculator showed it as " 4 ". This outcome might be justified by the fact that any experienced executive could not gather this number of factors (i.e. 31 variables) when evaluating a critical subjective organizational aspect.

In the Test Dataset, BITA level "2" and "4" have only one observation and three observations each. In this case, the available data is biased which causes deep learning to confront high distraction in making correct predictions resulting in the overall $89 \%$ accuracy rate using the model and $92 \%$ using the calculator. This bias is related mainly to lack of data as the algorithms seriously demand very large data sets to conduct training and subsequently testing. However, the author believes that these drawbacks and consequently the accuracy rates could be much higher if he becomes able to accomplish such requirement in future.

\section{Conclusions}

This paper seeks to examine the managerial significance of a model and a calculator recently produced relying on artificial intelligence in order to allow top executives to evaluate the maturity of strategic alignment based on objective criteria and indicators. The Diab BITA Model tested on the selected sample was successful in predicting the maturity level of BITA in $89 \%$ of the engaged organizations. $89 \%$ of senior executives were capable of subjectively estimating the degree to which their organizations' business strategy \& infrastructure integrate with their IT strategy \& infrastructure. Moreover, Diab 
Calculator achieved a 92\% accuracy rate which is almost equal to the rate of the model. Generally speaking, the model supports the ability of highly experienced executives in quantitatively judging a qualitative critical business concern, the BITA.

Managers require 15 minutes on average to rate the 31 variables completely while no one complained about wording, complexity, length or any other aspect of the model.

Finally, this paper serves as a further proof on the value of generated model and calculator which seriously form a practical validation for BITA measurement. It should likewise direct the author toward additional experiments for the model and calculator in a specific industry or another country with the cooperation of other researchers. Over and above, this study's output stimulates the author to work harder on the BITA calculator which they anticipate to constitute a milestone in the field of Strategic Business-IT alignment measurement.

\section{References}

[1] J. C. Henderson and N. Venkatraman, "Strategic alignment: Leveraging information technology for transforming organizations", IBM Systems Journal, 32(1), pp. 4-16, January 1993.

[2] What is the COBIT Framework: Benefits and Components, Available at: https://www.simplilearn.com/what-is- cobit-significance-and-frameworkrar309-article

Last updated on Jul 13, 2020.

[3] A. Oppermann, "What is deep learning and how does it work?", November 2019, Available at: https://towardsdatascience.com/what-isdeep-learning-and-how-does-it-work2ce44bb692ac

[4] M. Edmead, "IT Convergence Factor", October 2016, Available at: https://www.cio.com/article/3058875/me asuring-business-it convergence.html

[5] M. Iansiti and K. R. Lakhani, "Competing in the age of AI: Strategy and leadership when algorithms and networks run the world", Harvard Business School Publishing, January 2020.

[6] Business World Innovative Technologies, "What is Artificial Intelligence (AI) in business?", July 2020, Available at: https://www.businessworldit.com/ai/artifi cial-intelligence-in-business/

[7] Financial Times Future Forum, "The impact of AI on business and society", October 2020, Available at: https://www.ft.com/content/e082b01dfbd6-4ea5-a0d2-05bc5ad7176c

[8] I. Smeureanu and B. Diab, "Modeling the impact of Information System on organizational culture and Business-IT Strategic Alignment", Informatica Economica, 23(3), pp. 16-25, September 2019.

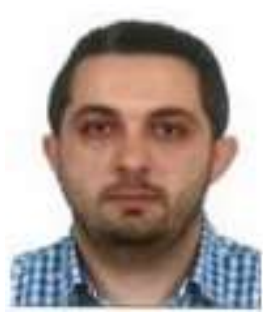

Bassel DIAB holds a bachelor degree in money, banking \& customs (2008) and a master degree in business administration (2017). He is a $\mathrm{PhD}$ candidate (3rd year) in Management Information Systems at the Bucharest University of Economic Studies (ASE) starting 2018, guided by professor Ion SMEUREANU. His work is mainly directed at the role of information system in supporting organizational communication, culture and performance. He has written a number of articles in this field. In professional career, he has progressed to occupy managerial positions like accounting manager and budgeting $\&$ cost control manager, and he is currently a finance manager. 
Appendix 1. - Diab Calculator (Diab, B., PhD 2020)

Please rank your answers on the following statements based on a 6-point Likert scale:

1: Totally disagree - 2: disagree - 3: Slightly disagree - 4: Slightly agree - 5: Agree - 6: Totally agree.

\begin{tabular}{|c|c|c|c|c|}
\hline Metrics & \begin{tabular}{|l|} 
Normalized \\
Importance \\
\end{tabular} & $\begin{array}{c}\text { Weight/ } \\
\text { unit }\end{array}$ & Actual & $\begin{array}{c}\text { Estimated } \\
\text { Importance } \\
\end{array}$ \\
\hline Strategic Business-IT Alignment & $28.94 \%$ & & & 0.29 \\
\hline IT understands the business environment of the organization (e.g. production cycle). & $100.00 \%$ & 0.012 & 6 & 0.07 \\
\hline Performance is ranked as high at organizational level. & $74.60 \%$ & 0.009 & 6 & 0.05 \\
\hline Strategic business planning is formally conducted with effective IT involvement. & $68.90 \%$ & 0.008 & 6 & 0.05 \\
\hline Top mgt considers significant IT and business decisions of equal impact in the org. & $50.90 \%$ & 0.006 & 6 & 0.04 \\
\hline Business executives understand the environment of IT (e.g. systems, resources). & $42.00 \%$ & 0.005 & 6 & 0.03 \\
\hline Strategic IT planning is formally conducted with business executives involvement. & $31.90 \%$ & 0.004 & 6 & 0.02 \\
\hline A committee, consisting of IT and business executives, exists to make shared decisions. & $26.10 \%$ & 0.004 & 6 & 0.02 \\
\hline Organizational Communication & $26.15 \%$ & & & 0.28 \\
\hline Modern communication technology is used to communicate time-sensitive info. & $62.80 \%$ & 0.007 & 6 & 0.04 \\
\hline IS facilitates the communication of org. culture elements (e.g. collaboration). & $61.50 \%$ & 0.008 & 6 & 0.05 \\
\hline IS ensures mgt a great amount of high quality info. from the surrounding community. & $54.10 \%$ & 0.006 & 6 & 0.04 \\
\hline Metrics & $\begin{array}{l}\text { Normalized } \\
\text { Importance } \\
\end{array}$ & $\begin{array}{c}\begin{array}{c}\text { Weight/ } \\
\text { unit }\end{array} \\
\end{array}$ & Actual & $\begin{array}{c}\text { Estimated } \\
\text { Importance }\end{array}$ \\
\hline IS supports executives in motivating employees to estimate the role of culture in business. & $42.80 \%$ & 0.005 & 6 & 0.03 \\
\hline IS contributes to abolishing distances when communicating sensitive info. & $42.50 \%$ & 0.005 & 6 & 0.03 \\
\hline IS leads to enhancing the quality of info shared among all levels of organizational members. & $40.40 \%$ & 0.005 & 6 & 0.03 \\
\hline IS increases the level of non-managerial staff's involvement in the decision making process. & $31.60 \%$ & 0.004 & 6 & 0.03 \\
\hline IS leads to underestimating the Middle Management Gap (i.e. mid-level managers). & $20.60 \%$ & 0.003 & 6 & 0.02 \\
\hline Organizational Culture & $20.87 \%$ & & & 0.18 \\
\hline IS (e.g. Cloud computing) enables employees to promoting their work-life balance. & $63.80 \%$ & 0.009 & 6 & 0.05 \\
\hline Management prioritizes information sharing over other organizational values. & $62.80 \%$ & 0.007 & 6 & 0.04 \\
\hline Management continuously makes enough investments in the IT infrastructure. & $36.30 \%$ & 0.004 & 6 & 0.02 \\
\hline Management sets a manual of rules and procedures in order to support the workflow of IS. & $32.00 \%$ & 0.004 & 6 & 0.03 \\
\hline IS supports executives in driving culture change. & $30.00 \%$ & 0.004 & 6 & 0.02 \\
\hline Mgt involves lower levels of org. members in the design, selection or development of IS. & $26.60 \%$ & 0.004 & 6 & 0.02 \\
\hline
\end{tabular}




\begin{tabular}{|c|c|c|c|c|}
\hline Metrics & $\begin{array}{l}\text { Normalized } \\
\text { Importance } \\
\end{array}$ & $\begin{array}{c}\text { Weight/ } \\
\text { unit }\end{array}$ & Actual & $\begin{array}{l}\text { Estimated } \\
\text { Importance } \\
\end{array}$ \\
\hline IS increases the readiness of the org. for institutionalization \& true corporate governance. & $24.80 \%$ & 0.004 & 6 & 0.02 \\
\hline IS supports the organization in adapting to fast changes in business environment. & $8.10 \%$ & 0.001 & 6 & 0.01 \\
\hline Organizational Performance & $24.04 \%$ & & & 0.25 \\
\hline IS directs the work of top executives toward strategic planning. & $55.70 \%$ & 0.007 & 6 & 0.04 \\
\hline IS provider is highly interested in a long lasting relationship with the organization. & $55.40 \%$ & 0.006 & 6 & 0.04 \\
\hline IS supports mgrs in maintaining coherent historical data for a better forecast. & $46.90 \%$ & 0.005 & 6 & 0.03 \\
\hline Training on IS provided by top mgt enables employees to optimizing its full capabilities. & $40.00 \%$ & 0.005 & 6 & 0.03 \\
\hline IS contributes to exceeding competitors with higher budgets (i.e. better mgt of resources). & $38.50 \%$ & 0.005 & 6 & 0.03 \\
\hline IS positively impacts the quality of managerial decisions. & $36.80 \%$ & 0.005 & 6 & 0.03 \\
\hline IS helps to at least retaining current customers (e.g. customized products \&/or services). & $31.80 \%$ & 0.003 & 6 & 0.02 \\
\hline \multirow[t]{2}{*}{ Org. makes benefit of IS (e.g. data science, machine learning) to promote its $R \& D$ activities. } & $22.50 \%$ & 0.003 & 6 & 0.02 \\
\hline & & & & 1.00 \\
\hline
\end{tabular}

Appendix 2. Diab Calculator Criteria (Diab, B., PhD 2020)

\begin{tabular}{|c|c|c|c|c|c|}
\hline Rating & Scale & Range & Outlook & Brief Description & Becommendations ftrend] \\
\hline A & a & $0.9 \times 1.0$ & Positive $\uparrow$ & Alignment maturity is extremely strong. & More strategic thinking (eg- keep Ir infrastructure up to-date, innovation, 8 \& o). \\
\hline B & 5 & $0.90 \cdot 0.89$ & Positive $\downarrow$ & Alignment maturity is strong. & Search for deficiencies and drawbacks (e.g. form or promote business-it cammittee). \\
\hline c & 4 & $0,60 \cdot 0.79$ & Under Review & Alignment maturity is adequate. & Work very fast to redefine ErTA, $\pi$ processes, etc, to avoid severe prohlems. \\
\hline D & 3 & $0.50-0.59$ & Risky & Alignement maturity is extremely wesk. & Set up metrics to reconstruct all business \& ir processes, educate senior staff on BIT, etc. \\
\hline$z$ & 2 & $0.00-0.49$ & N/A & Alignment does not exist. & Make fundarnental decisions e.g. hire new II directors/business executives \\
\hline \multicolumn{6}{|c|}{ non-blas factor is automatically computed and added to each one of the 4 sets of metrics } \\
\hline
\end{tabular}

